\title{
Estimation Of Reference Intervals For Plasma Amylase In Apparently Healthy Adults Of Southern Nigeria
}

\author{
${ }^{1, B . E . K A S I A ~, ~}{ }^{2,}$ C.G.ORLUWENE , ${ }^{1}$ U.B.A. MRAKPOR \\ MBBS; FMCPath B.Med. Sci; MBBS; FMCPath B.MLS \\ ${ }^{1,}$ Department of Chemical Pathology, Niger Delta University Teaching Hospital Okolobiri, \\ Bayelsa State, Nigeria \\ ${ }^{2,}$ Department of Chemical Pathology, University of Port Harcourt Teaching Hospital, \\ P. M. B. 6173; Port Harcourt, Rivers State, Nigeria.
}

\begin{abstract}
There is need for locally derived age-sex specific laboratory Reference Ranges (RR) for healthy Africans in southern Nigeria. Reference values from American and European population are used for African subjects despite previous studies showing significant differences Our aim was to establish clinical laboratory reference values for plasma amylase that can be used as a baseline for adult patients' management in southern Nigeria.This study was cross-sectional in design, carried out at the Niger Delta University Teaching Hospital, Okolobiri, Bayelsa State, Southern Nigeria. A total of 200 volunteers, M:F ratio (5.3:4.7) were selected. The exclusion criteria involved those free from chronic diseases like HIV and Hepatitis B. The piccolo express auto analyser assayed for plasma amylase.Reference ranges were constructed using non-parametric method to estimate $2.5^{\text {th }}$ and $97.5^{\text {th }}$ as the lower and upper limit respectively. Higher mean male than female values with no significant difference was observed in the reference ranges of plasma amylase. The RR of plasma amylase differed in the older age groups in both sexes. The developed reference values for amylase differed from American values used in our hospital.The differences in RR amongst population, age and sex justifies the need to interpret RR based on age, sex and population. It provides plasma amylase values to be used in southern Nigeria.
\end{abstract}

KEYWORDS: Age, Sex, Reference Ranges, Plasma amylase.

\section{INTRODUCTION}

Reference intervals are ranges of upper and lower limits of a given analyte which are used for a laboratory test to determine whether a disease is present or absent and to know if the patient is at risk from future disease states [1]. Plasma amylase is a measure of how much amylase (an enzyme mainly produced by the pancreas and salivary gland) is present in the blood stream. This test may be used as part of diagnostic workup for a patient with suspected abdominal trauma or kidney disease, as part of follow up to determine how well a patient is responding to treatment and to check for transplant rejection in patients who have received donor kidney. The test is minimally invasive and comes with low risk to the patient [2]. Elevated levels could be seen in inflamed kidneys, abdominal trauma, alcoholism, pancreatitis, cysts, gallstones etc. Laboratory interpretations of plasma amylase can only be made as either reduced, elevated or normal when compared with locally derived reference intervals.

Certain specified factors are needed when reference interval are to be established: (1)makeup of the reference population with reference to age, sex, genetic and socioeconomic factors;(2) the criteria used for including or excluding individuals from the reference sample groups;(3) the physiologic and environmental conditions by which the reference population was studied and sampled including time and date of collection, intake of food and drug, posture, smoking, degree of obesity, and stage of menstrual cycle;(4) the specimen collection procedure, including preparation of the individual; and (5) the analytical method used, including details of its precision and accuracy [3]. The IFCC also considers the terms (normal values, normal range, reference range or reference interval used interchangeably) to correspond to health associated (central 95\%) reference interval ${ }^{3}$.It is well known that reference intervals depend on many factors including type of instrument/reagents used, principle of method for test that is being performed, type of population served and strength of quality assurance practiced at the laboratory [4].

In the past, many hospital laboratories have either used the reference interval recommended by the instrument or test manufacturer or the values published in medical or laboratory textbooks. However, due to diversity of instrumentation, methodologies, reagents and population, it is important that large hospital 
laboratories determine their own reference intervals whereas, smaller laboratories due to limited resources should analyze fewer specimens (at least 20) and verify the reference intervals specified in the methods package insert, provided by the manufacturer [4Traditionally, normal reference ranges for clinical laboratory value have been obtained from the American and European population ${ }^{5}$. Moreover, there are variations in indices between these different populations and even amongst African ethnic groups [5]. Factors like genetics, dietary patterns, gender, age and environment are known to influence analytes. Thus, the use of normal lab values derived from external population can produce selective bias leading to exclusion of otherwise healthy persons. This creates a framework for incorrect patient management in routine clinical care. As a result of these differences, it is necessary to develop a reference range of local values for plasma amylase. Therefore, the aim of this study was to generate normal ranges of laboratory values for plasma amylase and age/sex variation in these values. This in addition was compared to RR previously reported. Relevant utilization of lab RR of this analyte is important in routine health assessment.

\section{SUBJECTS AND METHODS}

The study was approved by our institutional research and ethics committee after explaining the objectives and benefits of the study. Written and informed consent was sought and obtained from each participant before the study. A total of 200 apparently healthy staff of the Niger Delta University Teaching Hospital, Okolobiri, Bayelsa State, aged within 26-65years were recruited into the study. No participant was under any form of medication likely to influence the parameter under investigation. A general examination was carried out on each participant to rule out fever or jaundice. Their weight and height were determined using the secca scale. Body mass index (BMI) was calculated using the formula: weight in kilograms (kg) divided by the square of height in meters $\left(\mathrm{m}^{2}\right)$.Structured questionnaire was used in the collection of selected demographic data which includes age, sex, job description and history of alcohol abuse/medications like birth control pills, opiates or aspirin. Pregnant individuals and those with acute/chronic diseases were excluded from the study An overnight fast and the avoidance of caloric and caffeinated drink were advised, after which about $3 \mathrm{~mL}$ of venous blood was collected into a lithium heparin anticoagulant bottle. The blood specimen was centrifuged at 3500rpm and supernatant plasma collected. All analysis was done within $24 \mathrm{hrs}$ of sample collection at room temperature in batches of 40s for 1 week.

Plasma amylase was determined based on written SOP and maintained using the automated piccolo analyzer [6]. Calibration of test: The calibrator for automated systems was used. The system performed calibration automatically. Quality Assurance/Quality Control: Ensured accuracy and precision in test results. The system performed quality control automatically according to specifications in test. The pre analytical, analytical and post analytical phases of analysis were controlled throughout the study.

\section{STATISTICAL METHODS}

Data obtained was entered into Microsoft excel data base, compared and corrected for data errors, then imported into SPSS version 16.0. The data were visually inspected for extreme values and values for single parameters that appeared physiologically impossible were removed. Tables were drawn to show the normal distribution of analytes tested using the Kolmogorov-Smirnov method that provided mean and $95^{\text {th }}$ percentile. The $95^{\text {th }}$ percentile lower limit was defined as $2.5^{\text {th }}$ percentile and upper limit as $97.5^{\text {th }}$ percentile. The effects of different variables on the values of analytes were determined through independent sample T-test. A two-sided $\mathrm{p}$-value $<0.05$ was considered significant. Box and whisker plots showed that plasma amylase varied with age.

\section{RESULTS}

A total of 200 participants were enrolled in this study comprising of 106 males and 94 females bringing a M:F ratio to 5.3 :4.7. Reference range for plasma amylase was established for males and females with an age range of 26-65 years with mean age of 37.8 and 36.7 years for males and females respectively. Table 1 shows the socio demographic features of the participants which constituted high percentages of young and normal weight working population. The reference ranges were constructed using the $2.5^{\text {th }}$ and $97.5^{\text {th }}$ percentiles as lower and upper limits at 95\% confidence interval in accordance with CLSI (NCCLS,2000) [7] guideline for determining reference ranges. The means for males and females were statistically compared using the independent $t$-test, $\mathrm{p}<0.05$ was considered statistically significant. Table 2 shows sex specific reference ranges for plasma amylase based on the p-values for the difference between males and females. The reference values for the males was higher than that of the females but not statistically significant $(\mathrm{p}=0.320)$.

All studied participants were classified into 4 groups based on age intervals. The younger adults (26-35 and 3645yrs) comprised group 1 and 2 respectively whereas the middle aged (46-55 and 56-65yrs) constituted groups 3 and 4 respectively. The reference range difference between males and females for measured analytes was 
estimated by comparing the mean of each age group using independent t-test, where p-value $<0.05$ was considered significant.

The box and whisker plots shows elevated levels of plasma amylase with age in both males and females (figs 1 and 2) respectively.

\section{DISCUSSION}

The assessment of health of an individual is made on clinical grounds by medical history, physical examinations, laboratory tests and other special investigations using reliable reference data. The lack of reliable data in the developing countries is an important obstacle to effective management of health care and other social services. It is therefore necessary to develop reference data and improve information systems to aid decision makers and health care givers in planning, implementing and evaluating services [8]. The established reference ranges as documented in this study were higher in the males than females particularly as age progresses. The $2.5^{\text {th }}-97.5^{\text {th }}$ percentile being $44.8-158.8$ and $38.2-150.7 \mathrm{IU} / \mathrm{L}$ for males and females respectively. This was however not statistically significant $(\mathrm{p}>0.05)$. Similar sex related difference was reported by Henry R. [9] and Idonije et al [10] who described higher total plasma levels in normal healthy males when compared to their female counterparts. These differences however, were not sufficiently pronounced (significant) to propose gender related differences as suggested by Juan-Pereira et al [11] and S. Hepburn [12].

The reference samples were stratified according to sex and age in decades in order to discover any possible age related differences. No significant age related difference was seen in the younger age groups from this study which is comparable with previous studies by Henry R. [9], and Hohenwallner et al [13]. Nevertheless, the older age groups showed significant differences with the male values higher than that of their female counterparts, the possible explanation could be due to retained enzyme complexes associated with poor renal filtration and clearance with age. Racial differences have also been reported by previous studies $[14,15,16]$, stating that significant proportion of subjects of African and Asian origin have amylase activity above the reference interval different from the Caucasian population which is non-pathological. This index study value of 45-159U/L when compared to a Caucasian study of $28-100 \mathrm{U} / \mathrm{L}$ [14] and 14-97U/L [6] shows racial differences. This could be explained in terms of genetic/dietary differences among races. The s-type (amylase isoenzyme) has undergone duplication during evolution and many individuals have repeat of the gene (multiple tandem repeats) and the gene copy number is associated with apparent evolutionary exposure to high starch diets. Hence, Africans with predominantly starchy diets tend to have higher total amylase activity due to increase s-type amylase activity compared with those of Caucasian descent.

\section{CONCLUSION}

Generally, physiological factors vary from population to population due to diet, physical environment and socioeconomic factors. The reference values for plasma amylase determined in this study differed from the American value currently used to interpret laboratory results here in southern Nigeria. This justifies the need to use sex and age established reference values applicable to specific population rather than take from one population and apply to another. 
Table 1: Socio-demographic characteristics of the participants.

\begin{tabular}{|lcc|}
\hline Variable & Frequency $\mathbf{~ ( N ) ~}$ & Percentage (\%) \\
\hline Age intervals & 108 & 54 \\
$26-35$ & 48 & 24 \\
$36-45$ & 28 & 14 \\
$46-55$ & 14 & 7 \\
$56-65$ & & 53 \\
Gender & 106 & 47 \\
Males & 94 & 10 \\
Females & 20 & 18 \\
Occupation & 36 & 11 \\
Doctors & 22 & 51 \\
Nurses & 102 & \\
Lab. Scientist & 8 & 4 \\
Others & 70 & 35 \\
Body Mass index (BMI) & 66 & 33 \\
Underweight & 56 & 28 \\
Normal weight & & \\
Overweight & & \\
Obesity & & \\
& & \\
\end{tabular}

Table 2: Established Reference values for plasma Amylase in healthy adults.

\begin{tabular}{|c|c|c|c|c|c|c|c|c|}
\hline Variable & $\operatorname{Sex}(n)$ & Mean(SD) & p-value & Range & $2.5^{\text {th }}$ & $97.5^{\text {th }}$ & $95^{\text {th }}$ & $\mathbf{R R}$ \\
\hline \multirow[t]{2}{*}{ Amylase } & Male (106) & $92.1(35.0)$ & $0.320(\mathrm{NS})$ & $29-188$ & 44.8 & 158.8 & 154.2 & $44.8-158.8$ \\
\hline & Female (94) & $84.6(34.7)$ & & $27-179$ & 38.2 & 150.7 & 148.7 & $38.2-150.7$ \\
\hline \multicolumn{9}{|c|}{$\begin{array}{l}\text { Note: } \\
\text { RR = Reference range, } \\
p>0.05(N S)=\text { not significant, } \\
2.5^{\text {th }}, 95^{\text {th }}, 97.5^{\text {th }}=\text { values in percentile. }\end{array}$} \\
\hline
\end{tabular}


Table 3: Comparison of Reference Ranges for males and females in young and Older-aged adults.

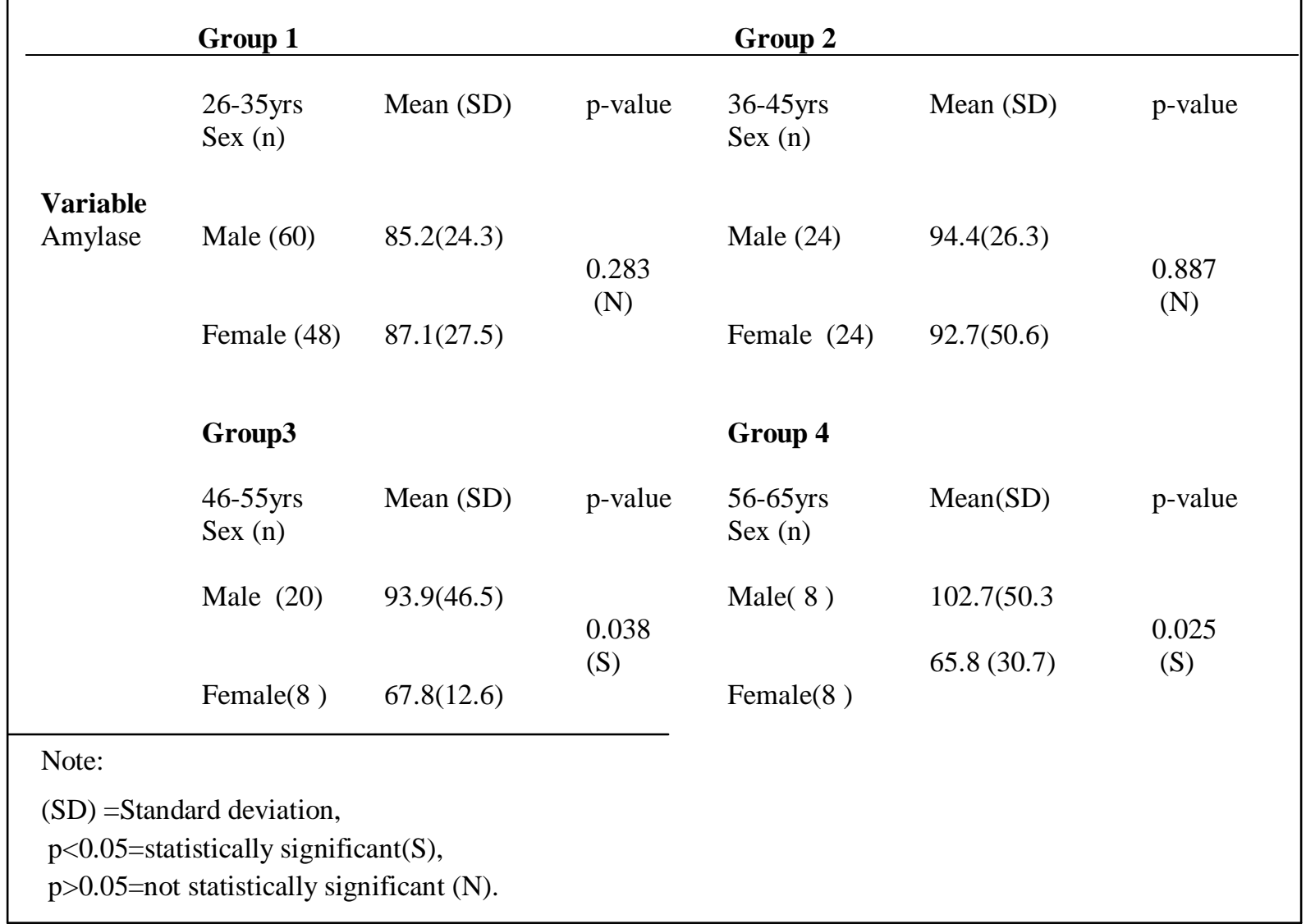

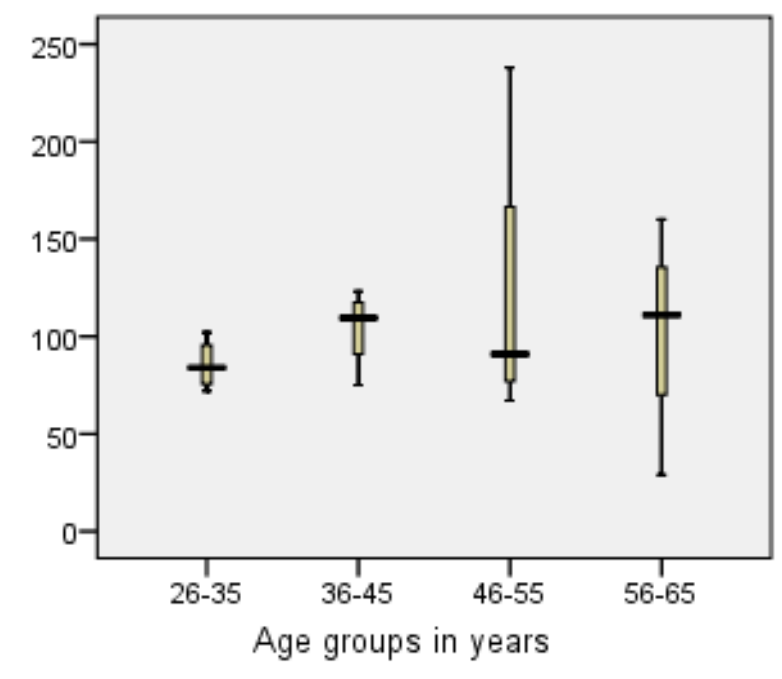

Fig. 1: Showing Plasma Amylase (U/L) variation within age groups in males

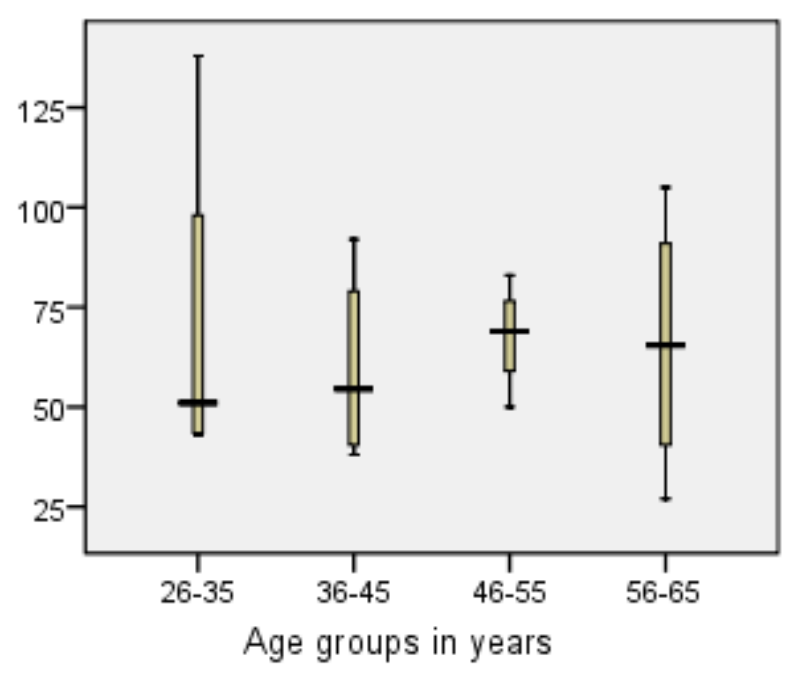

Fig. 2: Showing Plasma Amylase (U/L) variation within age groups in females 


\section{REFERENCES}

[1] Solberg HE. The International Federation of Clinical Chemistry (IFCC) Recommendation on estimation of refrence intervals. The RefVal program. Clin. Chem. Lab Med 42: 2004; 710-714.

[2] Smith SE, Shereen S. Wise Geek clear answers for common questions. What is plasma amylase? 2003-2013 Conjecture Corporation.

[3] Clinical and Laboratory Standard Institute (CLSI) C28 -A2: How to define and determine reference intervals in Clinical laboratory; Approved guideline.2nd ed., Villanova, Pa; CLSI, 2008.

[4] Solberg HE. IFCC Scientific Committee, clinical section, Expert Panel on theory of reference interval and international committee for standardization in Hematology(ICSH) Standing committee on Reference values. Approved Recommendation (1986) on the theory of reference values. Part 1. The concept of reference values. J Clin Chem Biochem; 25: 1987 337-342.

[5] Kibaya RS, Bautista CT, Sawe FK. Shaffer DN, Sateren WB et al. Reference ranges for the clinical laboratory derived from a rural population in Kericho, Kenya.2008;PLoS one 3:e3327.

[6] Piccolo Express Chemistry analyzer method manual 2006, Abaxis Inc.3240 Whipple Road, Union City, CA 94537, USA.

[7] National committee for Clinical Laboratory Standards (NCCLS) 2000. How to define, determine and utilize reference intervals in clinical laboratory. Approved guideline, NCCLS publications C28 -A2, Villanova PA, 12: 2000; 1-6.

[8] Adetokunbo OL., Herbert MG. Health Statistics and Information Systems. In Short Textbook of Public Health Medicine for the Tropics. Book power /ELST 4th Ed, 338 Euston Rd., London 2003:11-12.

[9] Henry RJ. Enzymes. In Clinical Chemistry, Principles and Techniques (Henry RJ, Cannon DC and Wilkelman J, eds) New York Harper and Row 1978, p949.

[10] Idonije OB, Festus OO, Agbebaku SO. A Comparative assessment of serum plasma and urine amylase levels in typhoid feverand HIV/AIDS patient. Pelagra Research library. Advances in applied science research 4(3): 2013; 16-22.

[11] Juan-Pereira, Andres-Moreno and Fuentes-Arderiu. Reference interval for serum alpha amylase. J.Clin Chem Biochem 23: 1985; $861-863$.

[12] Sophie Hepburn. Amylase, Name and Description of Analyte. Association for Clinical Biochemistry 2012; 1-8.

[13] Hohenwallner W, Hagela EO, Scholer A and Staber G, Ber Osterr Ges klin.Chem 6 1983; 101-112.

[14] Clave P et al. Enzymes. Clin Chem 41: 1995; 1129-1134.

[15] Carroll JK, Herrick B, Gipson T, Lee SP. Acute pancreatitis: diagnosis, prognosis and treatment. Am Fam Physician 75: 2007; $1513-1520$.

[16] Kiriyama S, Gabata T, Takada T et al. New diagnostic criteria of acute pancreatitis. J. Hepatobilary Pancreat Sci 17: 2010; $24-36$. 